\title{
Adequate antibiotic therapy prior to ICU admission in patients with severe sepsis and septic shock reduces hospital mortality

José Garnacho-Montero 1,2,3* , Antonio Gutiérrez-Pizarraya ${ }^{2,3,4}$, Ana Escoresca-Ortega', Esperanza Fernández-Delgado ${ }^{1}$ and José María López-Sánchez ${ }^{1}$

\begin{abstract}
Introduction: In patients with severe sepsis and septic shock as cause of Intensive Care Unit (ICU) admission, we analyze the impact on mortality of adequate antimicrobial therapy initiated before ICU admission.

Methods: We conducted a prospective observational study enrolling patients admitted to the ICU with severe sepsis or septic shock from January 2008 to September 2013. The primary end-point was in-hospital mortality. We considered two groups for comparisons: patients who received adequate antibiotic treatment before or after the admission to the ICU.

Results: A total of 926 septic patients were admitted to ICU, and 638 (68.8\%) had available microbiological isolation: 444 (69.6\%) received adequate empirical antimicrobial treatment prior to ICU and 194 (30.4\%) after admission. Global hospital mortality in patients that received treatment before ICU admission, between 0-6h ICU, 6-12h ICU, 12-24h ICU and after 24 hours since ICU admission were 31.3, 53.2, 57.1, 50 and 50.8\% ( $p<0.001)$. The multivariate analysis showed that urinary focus (odds ratio (OR) $0.20 ; 0.09-0.42 ; p<0.001$ ) and adequate treatment prior to ICU admission (OR 0.37; $0.24-0.56 ; p<0.001$ ) were protective factors whereas APACHE II score (OR 1.10; 1.07-1.14; $p<0.001$ ), septic shock (OR $2.47 ; 1.57-3.87 ; p<0.001)$, respiratory source (OR 1.91; 1.12-3.21; $p=0.016)$, cirrhosis (OR $3.74 ; 1.60-8.76 ; p=0.002$ ) and malignancy (OR 1.65; 1.02-2.70; $p=0.042$ ) were variables independently associated with in-hospital mortality. Adequate treatment prior to ICU was a protective factor for mortality in patients with severe sepsis $(n=236)$ or in septic shock ( $n=402)$.

Conclusions: The administration of adequate antimicrobial therapy before ICU admission is decisive for the survival of patients with severe sepsis and septic shock. Our efforts should be directed to assure the correct administration antibiotics before ICU admission in patients with sepsis.
\end{abstract}

\section{Introduction}

Acute management of patients with severe sepsis and septic shock is a very frequent task for ICU physicians. Initially, this management includes hemodynamic support and the administration of adequate antibiotics. Timely antibiotic administration is associated with decreased morbidity and mortality in critically ill patients

\footnotetext{
* Correspondence: jgarnachom@gmail.com

'Unidad Clínica de Cuidados Críticos, Hospital Universitario Virgen del Rocío/ CSIC/Universidad de Sevilla, Avda. Manuel Siurot s/n, 41013 Sevilla, Spain ${ }^{2}$ Instituto de Biomedicina de Sevilla (IBIS), Hospital Universitario Virgen del Rocío/CSIC/Universidad de Sevilla, Avda. Manuel Siurot s/n, 41013 Sevilla, Spain

Full list of author information is available at the end of the article
}

with septic shock [1-3]. Treatment protocols aiming at the rapid administration of adequate antibiotics are now considered a key element for improving the outcome of patients with septic shock [4]. Although the survival benefit associated with prompt antibiotic administration is clearly established for patients with septic shock, controversial results have been reported in patients with severe sepsis without shock $[3,5]$.

Although sepsis may occur in patients previously admitted to the ICU for other reasons, the great majority of sepsis episodes are diagnosed in hospital wards or in the emergency department (ED) and from these areas are transferred to the ICU. Several observational studies agree that only $10-30 \%$ of patients are located in the 
ICU when sepsis is identified [3, 6, 7]. It is noteworthy that acquisition of the infection before ICU admission has been identified as independently associated with early death in a large cohort of patients with sepsis [8].

The remaining episodes are diagnosed in the ED (50-60\%) and sepsis recognition is performed in other areas of the hospital (general wards or high-risk areas such as hematological or transplant units) in approximately 20-40 $\%$ of the cases $[3,6,7]$. The first antibiotic doses are therefore usually administered outside the ICU when culture results are not yet available [9].

Our aim is to analyze the impact on mortality of receiving adequate antimicrobial therapy before ICU admission compared with patients treated correctly after ICU admittance, in a cohort of patients admitted to the ICU for severe sepsis or septic shock. To better understand the burden of early adequate therapy on outcome, we performed this same analysis in severe sepsis and in septic shock separately.

\section{Materials and methods}

This study was performed in the ICU of Hospital Virgen del Rocío, a 40-bed medico-surgical unit in a large university hospital, from 1 January 2008 to 30 September 2013. The Institutional Review Board of Virgen del Rocío Hospital approved this protocol, waiving the need for informed consent given the observational design of this study.

All adult patients with severe sepsis or septic shock on admission to the ICU were included in a prospectively collected database. A previous study has been published with records included in this database [10]. The study included all patients in whom antimicrobial therapy was adequate before and after ICU admission and those who received adequate antibiotics only after being admitted to the ICU.

Based on the recommendations of the Surviving Sepsis Campaign [11], the hospital has a protocol for the management of severe sepsis and septic shock that includes initial therapy outside the ICU (fluid resuscitation, vasoactive drugs, source control, and empirical antimicrobial therapy). The protocol for antimicrobial therapy is updated annually by the Committee for Surveillance of Infections and Antimicrobial Use of Hospital Virgen del Rocío and is available on the hospital intranet.

The following variables were recorded: gender, age, chronic organ insufficiencies as defined by the Acute Physiology and Chronic Health Evaluation (APACHE) II scale, and other comorbidities (alcoholism, smoking habit, diabetes mellitus, noncure malignancy, and previous surgery) as defined previously [12]. At admission to the ICU, severity of the illness was evaluated by the APACHE II score and by the Sequential Organ Failure Assessment
(SOFA) scale that was also registered on admission and during the subsequent clinical course $[13,14]$.

In all patients, the following variables were also recorded: surgical or medical patient, nosocomial or communityacquired infection, clinical picture of sepsis (American College of Chest Physicians and the Society of Critical Care Medicine definitions), bacteremia, microbiologically documented infection, microorganism isolated, and appropriateness of empirical antibiotics. Candida infection was defined when it was recovered in blood culture or in any sterile sample. Isolation of Candida spp. in sputum or other respiratory samples was considered contamination. Bacteria included in the ESKAPE group (Enterococcus faecium, methicillin-resistant Staphylococcus aureus, Klebsiella pneumoniae, Acinetobacter baumannii, Pseudomonas aeruginosa, and Enterobacter species) which caused episodes were considered "difficult-to-treat" pathogens [15].

Antimicrobial therapy was considered adequate when the antibiotics prescribed covered all of the isolated pathogens (in blood and/or in the infection focus) and the dose and pattern of administration were in accordance with current standards. Patients were divided into two categories: patients in whom the antimicrobial therapy was adequate before ICU admission and those who received adequate antibiotics only after being admitted to the ICU.

Acquisition of a nosocomial infection was defined as the development of nosocomial pneumonia, primary bacteremia, and catheter-related bloodstream infection during the ICU stay following current definitions [16]. All patients were followed up until death or hospital discharge.

\section{Statistical analysis}

Discrete variables were expressed as counts (percentages) and continuous variables as means \pm standard deviation. The chi-square test or Fisher's exact test was used for categorical variables, and the Mann-Whitney $U$ test or Kruskal-Wallis test was used for continuous variables. To identify independent variables associated with in-hospital mortality, as well as assessing the impact of adequate antimicrobial therapy before ICU admission on the prognosis, we performed multivariate analysis by a binomial logistic regression model. To avoid spurious associations, variables entered into the regression models were those with a relationship in univariate analysis $(p<0.05)$, those with a plausible relationship with the dependent variable, or those that were clinically significant. Results are presented as odds ratio (OR) and 95\% confidence interval (CI). Potential explanatory variables were checked for colinearity before inclusion in the regression models using the tolerance and variance inflation factor. The threshold for statistical significance was defined as $p<0.05$. Data analysis was 
performed using SPSS for Windows 19.0 (SPSS, Inc., Chicago, IL, USA).

\section{Results}

The total cohort comprised 926 consecutive patients who were admitted to the ICU with the diagnosis of severe sepsis or septic shock. Sepsis was acquired in the community in $66.9 \%$ of the cases and an infection acquired in hospital wards was the cause of sepsis in 33.1 $\%$ of the episodes. The median time elapsed from triage in the ED to ICU admission was 5 hours.

Microbiological documentation was obtained in 641 cases $(69.2 \%)$. Three patients received adequate antimicrobial therapy before ICU admission but the regimen chosen once the patient was in the ICU was not correct. These patients were excluded; therefore 638 patients constitute the study cohort (402 with septic shock and 236 with severe sepsis). The median time elapsed from sepsis identification to the administration of adequate antimicrobial therapy was 4 hours.

The comparison of patients treated adequately before ICU admission and those who received adequate antimicrobial therapy once they were in the ICU is presented in Table 1. Inadequate therapy before ICU admission was prescribed more frequently in nosocomial sepsis than in community-acquired episodes (44.7 vs. $23 \% ; p<0.001)$. Notably, severity of illness at admission to the ICU was similar in both groups of patients. A higher proportion of episodes caused by difficult-totreat pathogens was found in patients who received adequate antibiotic only after being in the ICU. As expected, difficult-to-treat pathogens were more frequently isolated in nosocomial infections than in community-acquired episodes (36.6 vs. $16.6 \% ; p$ $<0.001)$.

Two-hundred and fourteen patients who were admitted from a general ward received the first dose of antibiotic in these wards (mainly in the internal medicine and general surgery wards). Otherwise, 390 patients with community-acquired infection received the first antibiotic in the ED and 23 patients received the first antibiotic in the operating room. One hundred and ninetyfour patients were treated inadequately before ICU admission. Only 11 of them had not received any antimicrobial agent before ICU admission. The remaining patients $(n=183)$ had received antibiotic treatment but it was judged inadequate based on culture results received once the patient was in the ICU. The uncovered pathogens $(n=189)$ in these 183 patients are presented in Table 2 (in six patients, two pathogens not initially covered were isolated).

Bivariate analysis of risk factors associated with hospital mortality is depicted in Table 3. Factors associated with fatality by multivariate analysis were APACHE
Table 1 Features of patients with adequate empirical antimicrobial therapy before or after ICU admission

\begin{tabular}{|c|c|c|c|}
\hline & $\begin{array}{l}\text { Before ICU (\%) } \\
(n=444)\end{array}$ & $\begin{array}{l}\text { Post ICU (\%) } \\
(n=194)\end{array}$ & $p$ value \\
\hline Age & $61(47-71)$ & $66(53-73)$ & 0.004 \\
\hline Gender (female) & $200(45)$ & $72(36.9)$ & 0.056 \\
\hline APACHE II score & $18(12-23)$ & $19(14-24)$ & 0.149 \\
\hline SOFA (median, IQR) & $7(4-10)$ & $8(5-10)$ & 0.170 \\
\hline Worst SOFA & $8(5-12)$ & $9(6-12)$ & 0.044 \\
\hline Surgical admission & $225(50.6)$ & $115(59)$ & 0.060 \\
\hline \multicolumn{4}{|l|}{ Acquisition } \\
\hline Community acquisition & $324(73)$ & $97(50)$ & \multirow[t]{2}{*}{$<0.001$} \\
\hline Hospital acquisition & $120(27)$ & $97(50)$ & \\
\hline \multicolumn{4}{|l|}{ Comorbidities } \\
\hline Diabetes & $102(23)$ & $46(23.6)$ & 0.865 \\
\hline COPD & $34(7.6)$ & $26(13.3)$ & 0.024 \\
\hline Cirrhosis & $24(5.4)$ & $16(8.2)$ & 0.183 \\
\hline Malignancy & $75(16.9)$ & $46(23.6)$ & 0.047 \\
\hline Chronic renal failure & $38(8.6)$ & $11(5.6)$ & 0.202 \\
\hline Immunosuppression & $68(15.3)$ & $33(16.9)$ & 0.608 \\
\hline Heart failure & $24(5.4)$ & $9(4.6)$ & 0.678 \\
\hline \multicolumn{4}{|l|}{ Sepsis source } \\
\hline Chest & $80(18)$ & $29(14.9)$ & 0.330 \\
\hline Urinary & $59(13.3)$ & $16(8.2)$ & 0.066 \\
\hline Abdomen & (45) & $106(54.4)$ & 0.030 \\
\hline Central nervous system & $18(4.1)$ & $2(1)$ & 0.043 \\
\hline Soft tissue & $51(11.5)$ & $19(9.7)$ & 0.516 \\
\hline Catheter & $15(3.4)$ & $8(4.1)$ & 0.651 \\
\hline Unidentified & $12(2.7)$ & $8(4.1)$ & 0.349 \\
\hline Positive blood culture & $224(50.5)$ & $97(49.7)$ & 0.869 \\
\hline \multicolumn{4}{|l|}{ Type of pathogen isolated } \\
\hline Gram-positive & $111(25)$ & $36(18.6)$ & 0.080 \\
\hline Gram-negative & $196(44.1)$ & $88(45.4)$ & 0.847 \\
\hline Anaerobic & $7(1.6)$ & $1(0.5)$ & 0.289 \\
\hline Fungus & $5(1.1)$ & $8(4.1)$ & 0.028 \\
\hline Polymicrobial & $120(27)$ & $59(30.4)$ & 0.304 \\
\hline Others & $5(1.1)$ & $2(1)$ & 0.141 \\
\hline Difficult-to-treat pathogens & $86(19.3)$ & $67(34.3)$ & $<0.001$ \\
\hline Septic shock & $281(63.3)$ & $121(62.4)$ & 0.825 \\
\hline Mechanical ventilation & $172(38.7)$ & $100(51.5)$ & 0.002 \\
\hline Nosocomial infection & $89(20.1)$ & 43/194 (22.2) & 0.580 \\
\hline ICU stay & $8(5-15)$ & $9(5-17)$ & 0.114 \\
\hline Hospital stay & $25(15-41)$ & $38(21-52)$ & 0.052 \\
\hline ICU mortality & $121(27.2)$ & $88(45.4)$ & $<0.001$ \\
\hline Hospital mortality & 139 (31.3) & $103(52.8)$ & $<0.001$ \\
\hline
\end{tabular}

APACHE Acute Physiology and Chronic Health Evaluation, COPD chronic obstructive pulmonary disease, CRBI catheter-related bloodstream infection, IQR interquartile range, SOFA Sequential Organ Failure Assessment 
Table 2 Pathogens isolated in patients with inadequate empirical therapy before ICU admission

\begin{tabular}{lll}
\hline & Focus & Bloodstream \\
\hline Gram-negative & & \\
Escherichia coli & $27(26.5)$ & $30(34.5)$ \\
Pseudomonas aeruginosa & $15(14.7)$ & $6(6.9)$ \\
Klebsiella pneumoniae & $11(10.8)$ & $9(10.3)$ \\
Acinetobacter baumannii & $7(6.9)$ & $4(4.6)$ \\
Proteus mirabilis & $2(2)$ & $1(1.1)$ \\
Enterobacter spp. & $5(4.9)$ & $4(4.6)$ \\
Gram-positive & & \\
Methicillin-resistant Staphylococcus aureus & $2(2)$ & $5(2.4)$ \\
Enterococcus spp. & $15(14.7)$ & $6(6.9)$ \\
Staphylococcus spp. & - & $3(3.4)$ \\
Fungi & & \\
Candida spp. & $8(7.8)$ & $11(12.6)$ \\
Others & $10(9.8)$ & $8(9.2)$ \\
Total & 102 & 87 \\
\hline
\end{tabular}

II score, cirrhosis, malignancy, pulmonary source, and septic shock, whereas urologic origin and adequate antimicrobial therapy before ICU admission were protective factors (Table 3). As shown in Fig. 1, ICU and hospital mortality rates were significantly lower in patients treated adequately before ICU admission but without difference regarding whether the adequate therapy was initiated in the first 6 hours in the ICU, between 6 and 12 hours, between 12 and 24 hours, or after more than 24 hours in the ICU $(p<0.001)$.

Four hundred and two patients presented septic shock at admission to the ICU. The mortality rate was $48.3 \%$. The bivariate analysis revealed that female gender, APACHE II, SOFA, cirrhosis, malignancy, and chest source of sepsis were significantly higher in nonsurvivors whereas a urinary source of sepsis and adequate antimicrobial therapy prior to admission to the ICU were more frequently administered in survivors than in nonsurvivors $(79.4$ vs. $60 \% ; p<0.001)$. In the multivariate analysis (Table 4), only four of these variables were independently associated with in-hospital mortality. Of note, adequate antimicrobial therapy before ICU admission was a protective factor for mortality (OR $0.40,95 \% \mathrm{CI}$ $0.24-0.65 ; p<0.001)$.

In the subgroup of patients with severe sepsis at admission to the ICU, 47 patients (19.9\%) died during hospitalization. Development of septic shock occurred less frequently in patients with adequate antimicrobial therapy prior to admission to the ICU than in those patients who received adequate antimicrobial therapy only after being admitted to the ICU (9.5 vs. $18 \%$; $p=0.002)$. The bivariate analysis revealed that age, APACHE II score, SOFA, chronic obstructive pulmonary disease (COPD), presence of immunosuppression, history of chronic heart failure, and development of nosocomial infection were significantly higher in nonsurvivors whereas adequate antimicrobial therapy prior to admission to the ICU was more frequently administered in survivors than in nonsurvivors (74.5 vs. $48.9 \% ; p<0.001)$. In the multivariate analysis, only five of these variables (Table 4 ) were independently associated with in-hospital mortality. Again, adequate antimicrobial therapy before ICU admission was included in the final regression model (OR $0.29,95 \%$ CI $0.13-0.63 ; p=0.002$ ).

\section{Discussion}

The present study demonstrates that, in septic patients, the administration of adequate empirical antimicrobial therapy before ICU admission is a protective factor for mortality after adjusting for numerous confounders. Importantly, mortality rates were significantly lower in patients treated adequately before ICU admission compared with those received adequate therapy even in the first 6 hours in the ICU. This advantage in terms of survival is present in patients with severe sepsis or in patients with septic shock.

Our model for in-hospital mortality includes, apart from the use of adequate antimicrobial therapy before ICU admission, variables frequently identified as predictors of fatality in large cohorts of sepsis, such as severity of illness, certain comorbidities, and the source of infection. Although the origin of sepsis has not been identified as a determinant of mortality [17], others have concluded that an urological source of sepsis is a protective factor and pneumonia is associated with a higher risk of death $[18,19]$.

Various studies have demonstrated the strong relationship existing between the delay in the administration of the first dose of antibiotics and mortality in patients with septic shock [1, 2]. The rate of inadequate empirical therapy in critically ill patients with sepsis depends primarily on the incidence of multidrug resistance pathogens and the adhesion to clinical guidelines, and ranges from 10 to almost $40 \%[18,20,21]$. In the present series, the great majority of the patients (98.3\%) had received at least one dose of antimicrobial before ICU admission although it was inadequate in $31 \%$ of them.

Emergency room utilization has increased over the last decade worldwide. The admission rate for sepsis from the ED has increased in comparison with admissions from hospital wards [22]. Outcomes for critically ill patients are influenced by whether or not optimal intensive support is delivered in a timely manner. Up to $20 \%$ of the patients with severe sepsis or septic shock do not fulfill diagnostic criteria in the first 3 hours in the ED $[23,24]$. In fact, sepsis is the predominant diagnostic 
Table 3 Bivariate and multivariate analyses of risk factors associated with hospital mortality

\begin{tabular}{|c|c|c|c|c|c|c|c|}
\hline & $\begin{array}{l}\text { Alive (\%) } \\
(n=397)\end{array}$ & $\begin{array}{l}\text { Death (\%) } \\
(n=241)\end{array}$ & $p$ value & $\begin{array}{l}\text { Unadjusted OR } \\
(95 \% \mathrm{Cl})\end{array}$ & $p$ value & $\begin{array}{l}\text { Adjusted OR } \\
(95 \% \mathrm{Cl})\end{array}$ & $p$ value \\
\hline$\overline{\text { Age }}$ & $60(45-71)$ & $65(55-73)$ & $<0.001$ & $1.01(1.00-1.25)$ & 0.050 & & \\
\hline Female gender & $176(44.2)$ & $96(39.8)$ & 0.248 & $0.81(0.54-1.21)$ & 0.314 & & \\
\hline APACHE \| score & $16(11-20)$ & $22(17-27)$ & $<0.001$ & $1.10(1.07-1.14)$ & $<0.001$ & $1.10(1.07-1.14)$ & $<0.001$ \\
\hline SOFA (median, IQR) & $6(4-9)$ & $10(7-12)$ & $<0.001$ & & & & \\
\hline Worst SOFA & $7(4-10)$ & $11(9-14)$ & $<0.001$ & & & & \\
\hline Surgical admission & $213(53.6)$ & $129(53.5)$ & 0.975 & & & & \\
\hline Hospital acquisition & $133(33.5)$ & $84(34.9)$ & 0.662 & & & & \\
\hline \multicolumn{8}{|l|}{ Comorbidities } \\
\hline Diabetes & $82(20.6)$ & $66(27.4)$ & 0.054 & & & & \\
\hline COPD & $30(7.6)$ & $30(12.4)$ & 0.040 & $1.10(0.57-2.12)$ & 0.755 & & \\
\hline Cirrhosis & $10(2.5)$ & $30(12.4)$ & $<0.001$ & $3.52(1.49-8.28)$ & 0.004 & $3.74(1.60-8.76)$ & 0.002 \\
\hline Malignancy & $60(15.1)$ & $61(25.3)$ & 0.002 & $1.50(0.90-2.48)$ & 0.112 & $1.65(1.02-2.70)$ & 0.042 \\
\hline Chronic renal failure & $26(6.5)$ & $23(9.5)$ & 0.173 & & & & \\
\hline Immunosuppression & $47(11.8)$ & $54(22.4)$ & $<0.001$ & $1.47(1.85-2.54)$ & 0.168 & & \\
\hline Heart failure & $18(4.5)$ & $15(6.2)$ & 0.350 & & & & \\
\hline \multicolumn{8}{|l|}{ Sepsis source } \\
\hline Chest & $55(13.8)$ & $54(22.4)$ & 0.006 & $1.84(1.07-3.15)$ & 0.026 & $1.91(1.12-3.21)$ & 0.016 \\
\hline Urinary & $64(16.1)$ & $11(4.5)$ & $<0.001$ & $0.20(0.09-0.42)$ & $<0.001$ & $0.20(0.09-0.42)$ & $<0.001$ \\
\hline Abdomen & $188(47.3)$ & $118(48.9)$ & 0.730 & & & & \\
\hline Central nervous system & $15(3.7)$ & $5(2.1)$ & 0.228 & & & & \\
\hline Soft tissue & $39(9.8)$ & $31(12.8)$ & 0.241 & & & & \\
\hline Catheter & $15(3.7)$ & $8(3.3)$ & 0.756 & & & & \\
\hline Unidentified & $11(2.7)$ & $9(3.7)$ & 0.504 & & & & \\
\hline Difficult-to-treat pathogens & $94(23.6)$ & $59(24.5)$ & 0.859 & & & & \\
\hline Positive blood culture & $196(49.3)$ & $125(51.9)$ & 0.576 & & & & \\
\hline Septic shock & $208(52.3)$ & $194(80.5)$ & $<0.001$ & $2.55(1.62-4.03)$ & $<0.001$ & $2.47(1.57-3.87)$ & $<0.001$ \\
\hline Nosocomial infection & $73(18.3)$ & $58(24.1)$ & 0.070 & & & & \\
\hline Prior ICU adequate antimicrobial therapy & $305(76.8)$ & $139(57.7)$ & $<0.001$ & $0.37(0.25-0.57)$ & $<0.001$ & $0.37(0.24-0.56)$ & $<0.001$ \\
\hline
\end{tabular}

APACHE Acute Physiology and Chronic Health Evaluation, $\mathrm{Cl}$ confidence interval, COPD chronic obstructive pulmonary disease, IQR interquartile range, OR odds ratio, SOFA Sequential Organ Failure Assessment

category in patients with delayed transfer from the ED to the ICU $[25,26]$.

Diverse studies have reported a significant delay in the administration of appropriate antibiotics in patients with severe sepsis and septic shock admitted to the ICU from the ED [27, 28]. Delays were greater for those in whom the diagnosis of sepsis was not considered initially. Sepsis can be difficult to diagnose because its clinical presentation mimics the symptoms of several other critical care conditions [29]. This fact may, at least in part, explain that only $60-80 \%$ of the patients received antibiotics in the ED in two recent retrospective studies carried out in the USA [30, 31].

In a small unicenter study, correct antibiotic coverage was prescribed by emergency physicians in $82 \%$ of the patients with culture-positive sepsis [9]. This was a retrospective study that found no impact on mortality considering the time elapsed from triage to the first dose of antibiotics in the ED. In contrast, a delay in the administration of appropriate antimicrobials from triage was independently associated with mortality. The use of broad-spectrum antibiotics was considered adequate in culture-negative episodes [32].

Antimicrobial resistance is an alarming clinical problem that is recognized internationally as one of the largest threats to human health. As in other series [9, 20], we observed that Gram-negative organisms comprise the most frequent cause for errors in empiric coverage. When antimicrobial therapy is initiated in the ED, multidrugresistant bacteria are covered very infrequently [30].

Approximately one-third of patients included in our series were transferred from the general ward to the 


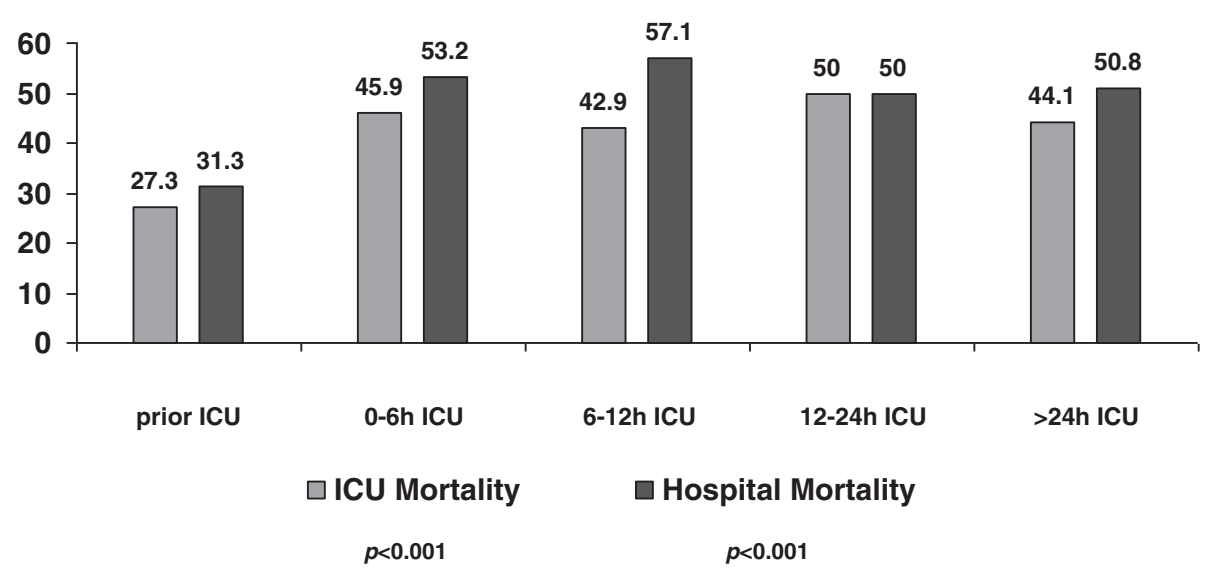

Fig. $1 \mathrm{ICU}$ and hospital mortality rates for the time range of adequate empirical antimicrobial therapy

ICU with a diagnosis of sepsis. Clinically significant delays in the process of care, including the administration of appropriate antibiotics, have been observed in patients with severe sepsis as it is in patients with septic shock in the general wards compared with those who presented septic shock when already admitted to the ICU [33]. In medical and surgical patients admitted to the general wards of a university-affiliated hospital, the median interval from the onset of severe sepsis or septic shock to the administration of antibiotic was 4 hours [34].

The impact on mortality of delayed antimicrobial therapy is not clearly established in patients with severe sepsis as it is in patients with septic shock $[1,2,5]$. Recently, a statically significant increment in the probability of death associated with the number of hours of delay for the first antibiotic administration was demonstrated in a large cohort of patients with severe sepsis and septic shock, although the subgroup of patients without shock was not evaluated specifically [3]. In a prospective study carried out in the EDs of three large hospitals, an increase in mortality with each hour delay to administration of antibiotics was demonstrated in patients with shock but not in patients with severe sepsis without development of shock [35]. In patients with community-acquired severe sepsis, a 6-hour delay or more in administration of the initial dose of antimicrobial was identified as an independent predictor of mortality and the authors could not demonstrate an independent impact on mortality during the previous hours [36].

We also found that progression to septic shock occurred more frequently in patients with severe sepsis who received inadequate antimicrobial therapy prior to ICU admission. Conversely, in hemodynamically stable patients presenting to the ED with sepsis, the use of appropriate antibiotics was similar in patients who developed shock within 72 hours and those who remained hemodynamically stable [35]. We and others have previously demonstrated that inadequate antimicrobial therapy is associated with a higher degree of organ dysfunction in the ICU (including cardiovascular system) than those who received adequate antimicrobial therapy [21, 37].

There are several limitations to this study. Management of septic patients involves infection control and organ support. Control of infection is achieved through prompt administration of adequate antibiotics and surgical drainage when necessary. We did not monitor timing of fluid resuscitation or source control. Prompt and

Table 4 Multivariate analysis of risk factors for hospital mortality in patients with severe sepsis and septic shock

\begin{tabular}{|c|c|c|c|c|}
\hline & \multicolumn{2}{|l|}{ Severe sepsis } & \multicolumn{2}{|l|}{ Septic shock } \\
\hline & Adjusted OR (95\% Cl) & $p$ value & Adjusted OR $(95 \% \mathrm{Cl})$ & $p$ value \\
\hline Age & $1.02(1.00-1.05)$ & 0.033 & & \\
\hline APACHE II score & $1.07(1.01-1.14)$ & 0.020 & $1.11(1.07-1.15)$ & $<0.001$ \\
\hline Cirrhosis & & & $4.49(1.55-13.04)$ & 0.006 \\
\hline Immunosuppression & $4.39(1.64-11.72)$ & 0.003 & & \\
\hline Urinary sepsis source & & & $0.11(0.05-0.27)$ & $<0.001$ \\
\hline Nosocomial infection & $6.53(2.74-15.55)$ & $<0.001$ & & \\
\hline Prior ICU adequate antimicrobial therapy & $0.29(0.13-0.63)$ & 0.002 & $0.40(0.24-0.65)$ & $<0.001$ \\
\hline
\end{tabular}


aggressive fluid resuscitation is associated with a higher survival rate in severe sepsis and septic shock [38]. Unfortunately, our study design did not include registering the time lag between the detection of the first signs or symptoms of sepsis and the administration of the first antibiotic dose, which hinders comparisons with series that analyzed the delay of administration of the first antibiotic dose.

\section{Conclusions}

We have demonstrated that the initiation of effective antimicrobial therapy before ICU admission is a critical determinant of outcome in patients with severe sepsis and septic shock. Our data highlight that the administration of adequate antibiotics once the patient is in the ICU is not sufficient because the mortality rate is significantly higher although the initiation of the correct antimicrobial therapy takes place in the first hours after ICU admission. Renewed efforts should be implemented to minimize the prescription of incorrect antimicrobials in septic patients, especially outside the ICU.

\section{Key messages}

- Approximately, one-third of the patients included in our series were transferred from the general ward to the ICU with a diagnosis of sepsis.

- The progression to septic shock occurred more frequently in patients with severe sepsis who received inadequate antimicrobial therapy prior to ICU admission.

- In septic patients, the administration of adequate empirical antimicrobial therapy before ICU admission is a protective factor of mortality.

\begin{abstract}
Abbreviations
APACHE: Acute Physiology and Chronic Health Evaluation; Cl: Confidence interval; COPD: Chronic obstructive pulmonary disease; ED: Emergency department; OR: Odds ratio; SOFA: Sequential Organ Failure Assessment..
\end{abstract}

Competing interests

The authors declare that they have no competing interests.

\section{Authors' contributions}

JG-M was responsible for the conception, fund raising, design and coordination of the study, made substantial contributions to data acquisition, analysis and interpretation, and drafted the manuscript. AG-P participated in the design and coordination of the study, performed the statistical analysis, and drafted the manuscript. AE-O participated in the study design and helped to draft the manuscript. EF-D made substantial contributions to the acquisition and interpretation of data. JML-S participated in the interpretation of data and was involved in drafting the manuscript. All authors read and approved the final manuscript.

\section{Acknowledgements}

This study was supported by Ministerio de Economía y Competitividad, Instituto de Salud Carlos III co-financed by the European Development Regional Fund "A Way to Achieve Europe", Spanish Network for the Research in Infectious Diseases (REIPI RD12/0015).

\section{Author details}

'Unidad Clínica de Cuidados Críticos, Hospital Universitario Virgen del Rocío/ CSIC/Universidad de Sevilla, Avda. Manuel Siurot s/n, 41013 Sevilla, Spain. ${ }^{2}$ Instituto de Biomedicina de Sevilla (IBIS), Hospital Universitario Virgen del Rocío/CSIC/Universidad de Sevilla, Avda. Manuel Siurot s/n, 41013 Sevilla, Spain. ${ }^{3}$ Red Española de Investigación en Patología infecciosa (REIPI), Hospital Universitario Virgen del Rocío/CSIC/Universidad de Sevilla, Avda. Manuel Siurot s/n, 41013 Sevilla, Spain. ${ }^{4}$ Unidad Clínica de Enfermedades Infecciosas, Microbiología y Medicina Preventiva, Hospital Universitario Virgen del Rocío, Avda. Manuel Siurot s/n, 41013 Sevilla, Spain.

Received: 2 June 2015 Accepted: 10 July 2015

Published online: 27 August 2015

\section{References}

1. Kumar A, Ellis P, Arabi Y, Roberts D, Light B, Parrillo JE, et al. Initiation of inappropriate antimicrobial therapy results in a fivefold reduction of survival in human septic shock. Chest. 2009;136:1237-48.

2. Kumar A, Roberts D, Wood KE, Light B, Parrillo JE, Sharma S, et al. Duration of hypotension before initiation of effective antimicrobial therapy is the critical determinant of survival in human septic shock. Crit Care Med. 2006;34:1589-96.

3. Ferrer R, Martin-Loeches I, Phillips G, Osborn TM, Townsend S, Dellinger RP, et al. Empiric antibiotic treatment reduces mortality in severe sepsis and septic shock from the first hour: results from a guideline-based performance improvement program. Crit Care Med. 2014;42:1749-55.

4. Nguyen HB, Rivers EP, Abrahamian FM, Moran GJ, Abraham E, Trzeciak S, et al. Severe sepsis and septic shock: review of the literature and emergency department management guidelines. Ann Emerg Med. 2006:48:28-54.

5. Puskarich MA, Trzeciak S, Shapiro NI, Arnold RC, Horton JM, Studnek JR, et al. Association between timing of antibiotic administration and mortality from septic shock in patients treated with a quantitative resuscitation protocol. Crit Care Med. 2011;39:2066-71.

6. Alberti C, Brun-Buisson C, Burchardi H, Martin C, Goodman S, Artigas A, et al Epidemiology of sepsis and infection in ICU patients from an international multicentre cohort study. Intensive Care Med. 2002;28:108-21.

7. Rohde JM, Odden AJ, Bonham C, Kuhn L, Malani PN, Chen LM, et al. The epidemiology of acute organ system dysfunction from severe sepsis outside of the intensive care unit. J Hosp Med. 2013;8:243-7.

8. Blanco J, Muriel-Bombín A, Sagredo V, Taboada F, Gandía F, Tamayo L, et al. Incidence, organ dysfunction and mortality in severe sepsis: a Spanish multicentre study. Crit Care. 2008;12:R158.

9. Capp R, Chang Y, Brown DFM. Effective antibiotic treatment prescribed by emergency physicians in patients admitted to the intensive care unit with severe sepsis or septic shock: where is the gap? J Emerg Med. 2011;41:573-80.

10. Garnacho-Montero J, Gutiérrez-Pizarraya A, Escoresca-Ortega A, CorciaPalomo Y, Fernández-Delgado E, Herrera-Melero I, et al. De-escalation of empirical therapy is associated with lower mortality in patients with severe sepsis and septic shock. Intensive Care Med. 2014;40:32-40.

11. Dellinger RP, Levy MM, Carlet JM, Bion J, Parker MM, Jaeschke R, et al. Surviving Sepsis Campaign: international guidelines for management of severe sepsis and septic shock: 2008. Crit Care Med. 2008;36:296-327.

12. Pittet D, Thiévent B, Wenzel RP, Li N, Gurman G, Suter PM. Importance of pre-existing co-morbidities for prognosis of septicemia in critically ill patients. Intensive Care Med. 1993;19:265-72.

13. Knaus WA, Draper EA, Wagner DP, Zimmerman JE. APACHE II: a severity of disease classification system. Crit Care Med. 1985;13:818-29.

14. Vincent JL, Moreno R, Takala J, Willatts S, De Mendonça A, Bruining H, et al. On behalf of the Working Group on Sepsis-Related Problems of the European Society of Intensive Care Medicine. Intensive Care Med. 1996;22:707-10.

15. Rice LB. Federal funding for the study of antimicrobial resistance in nosocomial pathogens: no ESKAPE. J Infect Dis. 2008;197:1079-81.

16. Calandra T, Cohen J. International Sepsis Forum Definition of Infection in the ICU Consensus Conference. The International Sepsis Forum consensus conference on definitions of infection in the intensive care unit. Crit Care Med. 2005;33:1538-48.

17. Zahar J-R, Timsit J-F, Garrouste-Orgeas M, Français A, Vesin A, Vesim A, et al. Outcomes in severe sepsis and patients with septic shock: pathogen 
species and infection sites are not associated with mortality. Crit Care Med. 2011;39:1886-95.

18. Garnacho-Montero J, Garcia-Garmendia JL, Barrero-Almodovar A, JimenezJimenez FJ, Perez-Paredes C, Ortiz-Leyba C. Impact of adequate empirical antibiotic therapy on the outcome of patients admitted to the intensive care unit with sepsis. Crit Care Med. 2003;31:2742-51.

19. Leligdowicz A, Dodek PM, Norena M, Wong H, Kumar A, Kumar A, et al. Association between source of infection and hospital mortality in patients who have septic shock. Am J Respir Crit Care Med. 2014;189:1204-13.

20. Zilberberg MD, Shorr AF, Micek ST, Vazquez-Guillamet C, Kollef MH. Multidrug resistance, inappropriate initial antibiotic therapy and mortality in Gram-negative severe sepsis and septic shock: a retrospective cohort study. Crit Care. 2014;18:596.

21. Ibrahim EH, Sherman G, Ward S, Fraser VJ, Kollef MH. The influence of inadequate antimicrobial treatment of bloodstream infections on patient outcomes in the ICU setting. Chest. 2000;118:146-55.

22. Azkárate I, Choperena G, Salas E, Sebastián R, Lara G, Elósegui I, et al. Epidemiology and prognostic factors in severe sepsis/septic shock. Evolution over six years. Med Intensiva. 2015 doi:10.1016/j.medin.2015.01.006.

23. Villar J, Clement JP, Stotts J, Linnen D, Rubin DJ, Thompson D, et al. Many emergency department patients with severe sepsis and septic shock do not meet diagnostic criteria within 3 hours of arrival. Ann Emerg Med. 2014:64:48-54.

24. Venkatesh AK, Avula U, Bartimus H, Reif J, Schmidt MJ, Powell ES. Time to antibiotics for septic shock: evaluating a proposed performance measure. Am J Emerg Med. 2013;31:680-3.

25. Chalfin DB, Trzeciak S, Likourezos A, Baumann BM, Dellinger RP. DELAY-ED study group. Impact of delayed transfer of critically ill patients from the emergency department to the intensive care unit. Crit Care Med. 2007;35:1477-83

26. Louriz M, Abidi K, Akkaoui M, Madani N, Chater K, Belayachi J, et al. Determinants and outcomes associated with decisions to deny or to delay intensive care unit admission in Morocco. Intensive Care Med. 2012;38:830-7.

27. Francis $M$, Rich $T$, Williamson $T$, Peterson D. Effect of an emergency department sepsis protocol on time to antibiotics in severe sepsis. CJEM. 2010;12:303-10.

28. Cullen M, Fogg T, Delaney A. Timing of appropriate antibiotics in patients with septic shock: a retrospective cohort study. Emerg Med Australas. 2013;25:308-15.

29. Heffner AC, Horton JM, Marchick MR, Jones AE. Etiology of illness in patients with severe sepsis admitted to the hospital from the emergency department. Clin Infect Dis. 2010;50:814-20.

30. Filbin MR, Arias SA, Camargo CA, Barche A, Pallin DJ. Sepsis visits and antibiotic utilization in U.S. emergency departments. Crit Care Med. 2014;42:528-35.

31. Capp R, Horton CL, Takhar SS, Ginde AA, Peak DA, Zane R, et al. Predictors of patients who present to the emergency department with sepsis and progress to septic shock between 4 and 48 hours of emergency department arrival. Crit Care Med. 2015;43:983-8.

32. Gaieski DF, Mikkelsen ME, Band RA, Pines JM, Massone R, Furia FF, et al. Impact of time to antibiotics on survival in patients with severe sepsis or septic shock in whom early goal-directed therapy was initiated in the emergency department. Crit Care Med. 2010;38:1045-53.

33. Lundberg JS, Perl TM, Wiblin T, Costigan MD, Dawson J, Nettleman MD, et al. Septic shock: an analysis of outcomes for patients with onset on hospital wards versus intensive care units. Crit Care Med. 1998;26:1020-4.

34. Mok K, Christian MD, Nelson S, Burry L. Time to administration of antibiotics among inpatients with severe sepsis or septic shock. Can J Hosp Pharm. 2014;67:213-9

35. Glickman SW, Cairns CB, Otero RM, Woods CW, Tsalik EL, Langley RJ, et al. Disease progression in hemodynamically stable patients presenting to the emergency department with sepsis. Acad Emerg Med. 2010;17:383-90

36. Nygård ST, Langeland N, Flaatten HK, Fanebust R, Haugen O, Skrede S. Aetiology, antimicrobial therapy and outcome of patients with community acquired severe sepsis: a prospective study in a Norwegian university hospital. BMC Infect Dis. 2014;14:121.
37. Garnacho-Montero J, Ortiz-Leyba C, Herrera-Melero I, Aldabó-Pallás T, Cayuela-Dominguez A, Marquez-Vacaro JA, et al. Mortality and morbidity attributable to inadequate empirical antimicrobial therapy in patients admitted to the ICU with sepsis: a matched cohort study. J Antimicrob Chemother. 2008;61:436-41.

38. Lee SJ, Ramar K, Park JG, Gajic O, Li G, Kashyap R. Increased fluid administration in the first three hours of sepsis resuscitation is associated with reduced mortality: a retrospective cohort study. Chest. 2014;146:908-15.

\section{Submit your next manuscript to BioMed Central and take full advantage of:}

- Convenient online submission

- Thorough peer review

- No space constraints or color figure charges

- Immediate publication on acceptance

- Inclusion in PubMed, CAS, Scopus and Google Scholar

- Research which is freely available for redistribution 